\title{
Symmetry Energy Effects on the Mixed Hadron-Quark Phase at High Baryon Density
}

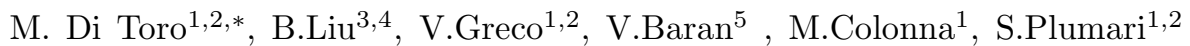 \\ 1 Laboratori Nazionali del Sud INFN, I-95123 Catania, Italy \\ 2 Physics and Astronomy Dept., University of Catania \\ 3 IHEP, Chinese Academy of Sciences, Beijing, China \\ 4 Theoretical Physics Center for Scientific Facilities, \\ Chinese Academy of Sciences, 100049 Beijing, China \\ 5 Physics Faculty, Univ. of Bucharest and NIPNE-HH, Romania \\ * email: ditoro@lns.infn.it
}

\begin{abstract}
The phase transition of hadronic to quark matter at high baryon and isospin density is analyzed. Relativistic mean field models are used to describe hadronic matter, and the MIT bag model is adopted for quark matter. The boundaries of the mixed phase and the related critical points for symmetric and asymmetric matter are obtained. Due to the different symmetry term in the two phases, isospin effects appear to be rather significant.

With increasing isospin asymmetry the binodal transition line of the $\left(T, \rho_{B}\right)$ diagram is lowered to a region accessible through heavy ion collisions in the energy range of the new planned facilities, e.g. the FAIR/NICA projects. Some observable effects are suggested, in particular an Isospin Distillation mechanism with a more isospin asymmetric quark phase, to be seen in charged meson yield ratios, and an onset of quark number scaling of the meson/baryon elliptic flows .

The presented isospin effects on the mixed phase appear to be robust with respect to even large variations of the poorly known symmetry term at high baryon density in the hadron phase. The dependence of the results on a suitable treatment of isospin contributions in effective QCD Lagrangian approaches, at the level of explicit isovector parts and/or quark condensates, is finally discussed.
\end{abstract}

PACS numbers: 21.65.Mn,21.65Ef,25.75.Nq,05.70.Ce

Keywords: Nuclear Matter at High Baryon Density; Symmetry Energy; Deconfinement Transition; Critical End Point; Effective QCD Lagrangians

\section{INTRODUCTION}

Several suggestions are already present about the possibility of interesting isospin effects on the transition to a mixed hadron-quark phase at high baryon density 1,3$]$. This seems to be a very appealing physics program for the new facilities, FAIR at GSI-Darmstadt [4] and NICA at JINR-Dubna [5], where heavy ion beams (even unstable, with large isospin asymmetry) will be available with good intensities in the 1-30 AGeV energy region.

The weak point of those predictions is the lack of a reliable Equation of State (EoS) that can describe in a consistent way the two phases, hadronic and deconfined, at high baryon density.

In particular all the Two-EoS models obviously cannot reproduce continous transitions, like second order phase transitions or cross-overs. However they can be useful to check if we can have a first-order transition at lower temperatures. In the latter case, while we cannot localize the corresponding Critical End Point, we can study with some confidence the properties of the mixed phase region if realistic effective interactions in the two phases are used. Such discussion will also lead to a strong motivation to work on more refined effective theories for a strong interacting matter. The aim of our paper is just to show new results, on the dependence on the EoS choices in the two phases and on possible observables, that would further stimulate the search in the field, in theory as well as in experiment.

Isospin effects on the transition are ruled by the sym- metry term in the two phases. For the hadronic side in all the Two-EoS approaches, so far mostly applied to develop hybrid models for neutron stars, a rather strong density dependence of the symmetry energy has been used [1 $3,6-11]$. This point however is still open mainly due to the present lack of good data for isospin effects on Heavy Ion Collisions at intermediate energies, in particular on collective flows and particle productions [12 17. Here we extend our study also to cases with a much softer hadronic symmetry term in order to check the "robustness" of the expected isospin effects.

For the quark matter MIT-Bag [18], in refs. [1 3 , 6 8], or Nambu-Jona Lasinio (NJL) [19, 20], in refs. [911], models have been adopted, always without explicit isospin dependent contributions. Here we also mainly used standard MIT-Bag models, but we also discuss the consequence of some isospin effects in NJL approaches and possible indirect corrections due to the color-pairing residual interaction [21].

We finally like to note that the isospin dependence predictions can be also used in the opposite way: if we see such isospin effects on the sensitive observables suggested here we can get more confidence on the reliability of the used EoS's in the two phases.

This is the plan of the paper. In Sect.I we present a simple motivation for a first order transition with isospin effects. In Sect.II the procedure to construct the "binodal surface" from the Gibbs conditions is presented, with particular attention to the physical interpretation of the observed end point. Properties of the mixed phase 


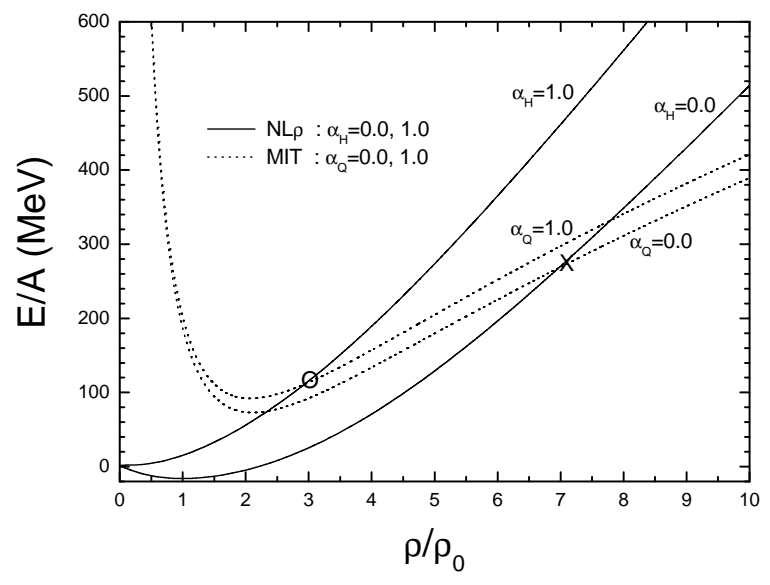

FIG. 1: Zero temperature EoS of Symmetric/Neutron Matter: Hadron $(N L \rho)$, solid lines, vs. Quark (MIT-Bag), dashed lines. $\alpha_{H, Q}$ represent the isospin asymmetry parameters respectively of the hadron,quark matter: $\alpha_{H, Q}=0$, Symmetric Matter; $\alpha_{H, Q}=1$, Neutron Matter.

are evaluated in Sect.III, using different density dependent symmetry terms for the hadron sector. Sect.IV is devoted to the introduction of isospin contributions in the quark effective EoS. Sensitive observables in collisions of neutron-rich ions at intermediate energies are suggested in Sect.V, with relative perspectives. Finally in Appendix A we present details of the used effective hadron interactions and in Appendix B the isospin dependent extension of the NJL model discussed in the paper.

\section{WHY A FIRST-ORDER TRANSITION WITH ISOSPIN EFFECTS?}

The main qualitative argument in favor of a first order hadron-quark transition at high density and low temperature, with noticeable isospin effects, can be derived from the Fig [1. Here we compare typical Equations of State for Hadron (Nucleon) and Quark Matter, at zero temperature, for symmetric $\left(\alpha \equiv\left(\rho_{n}-\rho_{p}\right) / \rho_{B} \equiv-\rho_{3} / \rho_{B}=0.0\right)$ and neutron matter $(\alpha=1.0)$, where $\rho_{n, p}$ are the neutron/proton densities and $\rho_{B}=\rho_{n}+\rho_{p}$ the total baryon density.

In this first simple calculation, a kind of "homework", for the hadron part we use a Relativistic Mean Field (RMF) EoS ([12, 22, 23] ) with non-linear terms and an effective $\rho$-meson coupling for the isovector part, largely used to study isospin effects in relativistic heavy ion collisions [3, 12]. However in the paper we will probe several effective hadron interactions to check the "robustness" of the observed symmetry energy effects. In order to keep a smooth flow of the physics points in the discus- sion, details about the adopted effective nucleon-meson Lagrangians are presented in the Appendix A.

The energy density and the pressure for the quark phase are given by the MIT Bag model [18] (two-flavor case) and read, respectively:

$$
\begin{aligned}
& \epsilon=3 \times 2 \sum_{q=u, d} \int \frac{\mathrm{d}^{3} k}{(2 \pi)^{3}} \sqrt{k^{2}+m_{q}^{2}}\left(f_{q}+\bar{f}_{q}\right)+B, \\
& P=\frac{3 \times 2}{3} \sum_{q=u, d} \int \frac{\mathrm{d}^{3} k}{(2 \pi)^{3}} \frac{k^{2}}{\sqrt{k^{2}+m_{q}^{2}}}\left(f_{q}+\bar{f}_{q}\right)-B,
\end{aligned}
$$

where B denotes the bag constant (the bag pressure), taken as a rather standard value from the hadron spectra $\left(B=85.7 \mathrm{MeV} \mathrm{fm}^{-3}\right.$, no density dependence), $m_{q}$ are the quark masses $\left(m_{u}=m_{d}=5.5 \mathrm{MeV}\right.$ choice), and $f_{q}$, $\bar{f}_{q}$ represent the Fermi distribution functions for quarks and anti-quarks. The quark number density is given by

$$
\rho_{i}=<q_{i}^{+} q_{i}>=3 \times 2 \int \frac{\mathrm{d}^{3} k}{(2 \pi)^{3}}\left(f_{i}-\bar{f}_{i}\right), \quad i=u, d .
$$

The transition to the more repulsive quark matter will appear around the crossing points of the two EoS. We see that such crossing for symmetric matter $\left(\alpha_{H}=\alpha_{Q}=\right.$ $0.0)$ is located at rather high density, $\rho_{B} \simeq 7 \rho_{0}$, while for pure neutron matter $\left(\alpha_{H}=\alpha_{Q}=1.0\right)$ it is moving down to about three times $\rho_{0}$. Of course the Fig.1 represents just a simple energetic argument to support the hadronquark transition to occur at lower baryon densities for more isospin asymmetric matter. In the rest of the paper we will rigourously consider the case of a first order phase transition in the Gibbs frame for a system with two conserved charges (baryon and isospin), in order to derive more detailed results. Since the first order phase transition presents a jump in the energy, we can expect the mixed phase to start at densities even before the crossing points of the Fig.1. The lower boundary then can be predicted at relatively low baryon densities for asymmetric matter, likely reached in relativistic heavy ion collisions. Moreover this point is certainly of interest for the structure of the crust and the inner core of neutron stars, e.g. see refs. 6 11] and the review 24]. We remark that in ref. [6] similar results are obtained with rather different hadronic approaches, the RMF and the non-relativistic Brueckner-Hartree-Fock (BHF) theory.

We finally note that the above conclusions are rather independent on the isoscalar part of the used Hadron EoS at high density, that is chosen to be rather soft in agreement with collective flow and kaon production data [25, 26].

In the used Bag Model no residual gluon interactions, the $\alpha_{s}$-strong coupling parameter, are included. We remark that this in fact would enhance the above effect, in the direction of overall lower transition densities, since it 
represents an attractive correction for a fixed B-constant, see [27]. A reduction of the Bag-constant with increasing baryon density, as suggested by various models, see ref. [6], will also go in the direction of an "earlier" (lower density) transition, as already seen in ref.[2]. At variance, the presence of explicit isovector contributions in the quark phase could play an important role, as shown in the following also for other isospin properties inside the mixed phase.

\section{ISOSPIN EFFECTS ON THE MIXED PHASE}

We can study in detail the isospin dependence of the transition densities [1 $[3]$. The structure of the mixed phase is obtained by imposing the Gibbs conditions [28] for chemical potentials and pressure and by requiring the conservation of the total baryon and isospin densities:

$$
\begin{aligned}
& \mu_{B}^{H}\left(\rho_{B}^{H}, \rho_{3}^{H}, T\right)=\mu_{B}^{Q}\left(\rho_{B}^{Q}, \rho_{3}^{Q}, T\right), \\
& \mu_{3}^{H}\left(\rho_{B}^{H}, \rho_{3}^{H}, T\right)=\mu_{3}^{Q}\left(\rho_{B}^{Q}, \rho_{3}^{Q}, T\right), \\
& P^{H}(T)\left(\rho_{B}^{H}, \rho_{3}^{H}, T\right)=P^{Q}(T)\left(\rho_{B}^{Q}, \rho_{3}^{Q}, T\right), \\
& \rho_{B}=(1-\chi) \rho_{B}^{H}+\chi \rho_{B}^{Q}, \\
& \rho_{3}=(1-\chi) \rho_{3}^{H}+\chi \rho_{3}^{Q},
\end{aligned}
$$

where $\chi$ is the fraction of quark matter in the mixed phase and $\mathrm{T}$ is the temperature.

The consistent definitions for the densities and chemical potentials in the two phases are given by :

$$
\begin{aligned}
& \rho_{B}^{H}=\rho_{p}+\rho_{n}, \quad \rho_{3}^{H}=\rho_{p}-\rho_{n}, \\
& \mu_{B}^{H}=\frac{\mu_{p}+\mu_{n}}{2}, \quad \mu_{3}^{H}=\frac{\mu_{p}-\mu_{n}}{2},
\end{aligned}
$$

for the Hadron Phase and

$$
\begin{aligned}
\rho_{B}^{Q}=\frac{1}{3}\left(\rho_{u}+\rho_{d}\right), & \rho_{3}^{Q}=\rho_{u}-\rho_{d}, \\
\mu_{B}^{Q}=\frac{3}{2}\left(\mu_{u}+\mu_{d}\right), & \mu_{3}^{Q}=\frac{\mu_{u}-\mu_{d}}{2},
\end{aligned}
$$

for the Quark Phase.

The related asymmetry parameters are:

$$
\alpha^{H} \equiv-\frac{\rho_{3}^{H}}{\rho_{B}^{H}}=\frac{\rho_{n}-\rho_{p}}{\rho_{n}+\rho_{p}}, \quad \alpha^{Q} \equiv-\frac{\rho_{3}^{Q}}{\rho_{B}^{Q}}=3 \frac{\rho_{d}-\rho_{u}}{\rho_{d}+\rho_{u}} .
$$

Nucleon and quark chemical potentials, as well as the pressures in the two phases, are directly derived from the respective EoS.

In this way we get the binodal surface which gives the phase coexistence region in the $\left(T, \rho_{B}, \rho_{3}\right)$ space. For a fixed value of the total asymmetry $\alpha_{T}=-\rho_{3} / \rho_{B}$ we will study the boundaries of the mixed phase region in the $\left(T, \rho_{B}\right)$ plane. Since in general the charge chemical potential is related to the symmetry term of the EoS, [12], $\mu_{3}=2 E_{\text {sym }}\left(\rho_{B}\right) \frac{\rho_{3}}{\rho_{B}}$, we expect critical and transition densities rather sensitive to the isovector channel in the two phases.

In the hadron sector we will use the Non-Linear Relativistic Mean Field models, [3, 12, 23], with different structure of the isovector part, already tested to describe the isospin dependence of collective flows and meson production for heavy ion collisions at intermediate energies, 29 31]. We will refer to these different Iso-Lagrangians as: i) $N L$, where no isovector meson is included and the symmetry term is only given by the kinetic Fermi contribution, ii) $N L \rho$ when the interaction contribution of an isovector-vector meson is considered and finally iii) $N L \rho \delta$ where also the contribution of an isovector-scalar meson is accounted for. See details in Appendix A1 and refs. $3,12,23$.

We will look at the effect on the hadron-quark transition of the different stiffness of the symmetry term at high baryon densities in the different parametrizations. As clearly shown in Appendix A1, where a rather transparent form for the density dependence of the symmetry energy in RMF approaches is discussed, the potential part of the symmetry term will be proportional to the baryon density in the $N L \rho$ choice and even stiffer in the $N L \rho \delta$ case.

We are well aware that there are several uncertainties on the stiffness of the symmetry energy at high baryon density, mainly due to the lack of suitable data, see the reviews $[12,14]$. Therefore in the next Section we will show also results with effective hadron interactions based on RMF models with density dependent meson-nucleon couplings ( $D D R H$ forces, Appendix A2) that present much softer symmetry terms at high baryon density. In this way we can directly check the "stability" of the observed isospin effects on the mixed phase.

As already mentioned, in the quark phase we use the MIT-Bag Model, where the symmetry term is only given by the Fermi contribution. The Bag parameter B is fixed for each baryon density to a constant, rather standard, value $B^{1 / 4}=160 \mathrm{MeV}$, corresponding to a Bag Pressure of $85.7 \mathrm{MeV} \mathrm{fm}^{-3}$.

In general for each effective interactive Lagrangian we can simulate the solution of the highly non-linear system of Eqs.(4), via an iterative minimization procedure, in order to determine the binodal boundaries.

A relatively simple calculation can be performed at zero temperature. The isospin effect (asymmetry dependence) on the Lower $(\chi=0.0)$ and Upper $(\chi=1.0)$ transition densities of the Mixed Phase are shown in Fig 2 for various choices of the Hadron EoS. The effect of a larger repulsion of the symmetry energy in the hadron sector, from $N L$ to $N L \rho$ and to $N L \rho \delta$, is clearly evident on the lower boundary with a sharp decrease of the transition density even at relatively low asymmetries.

Typical results for isospin effects on the whole binodal "surface" are presented in Fig 3 for symmetric and asymmetric matter. For the hadron part we have started from 


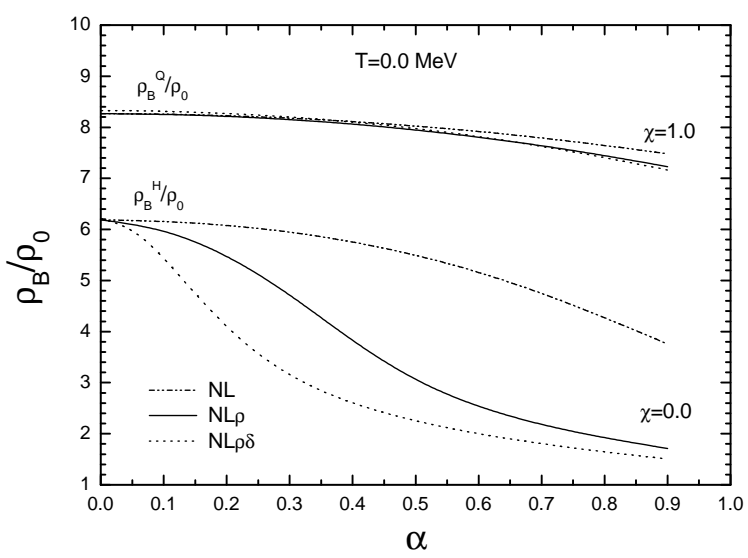

FIG. 2: Dependence on the Hadron Symmetry Energy of the Lower $(\chi=0.0)$ and Upper $(\chi=1.0)$ Boundaries of the Mixed Phase, at zero temperature, vs. the asymmetry parameter. Quark EoS: MIT bag model with $B^{1 / 4}=160 \mathrm{MeV}$.

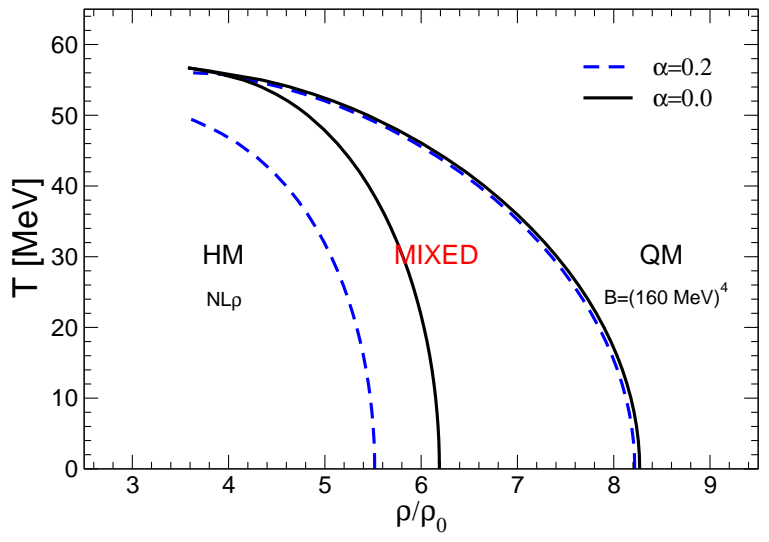

FIG. 3: Binodal surface for symmetric $(\alpha=0.0)$ and asymmetric $(\alpha=0.2)$ matter. Hadron EoS from $N L \rho$ interaction. Quark EoS: $M I T$ bag model with $B^{1 / 4}=160 \mathrm{MeV}$.

a $N L \rho$ effective Lagrangian very close to other widely used relativistic effective models, e.g. see the GM3 of ref. 32] and the NL3 interaction of P.Ring and collaborators [33], which has also given good nuclear structure results, even for exotic nuclei.

As expected, the lower boundary of the mixed phase is mostly affected by isospin effects. In spite of the relatively small total asymmetry, $\alpha=0.2$, we clearly observe in Fig 3 a shift to the left of the first transition boundary, in particular at low temperature.

In the symmetric matter case the mixed phase is evaluated from the simpler Maxwell conditions. The re-
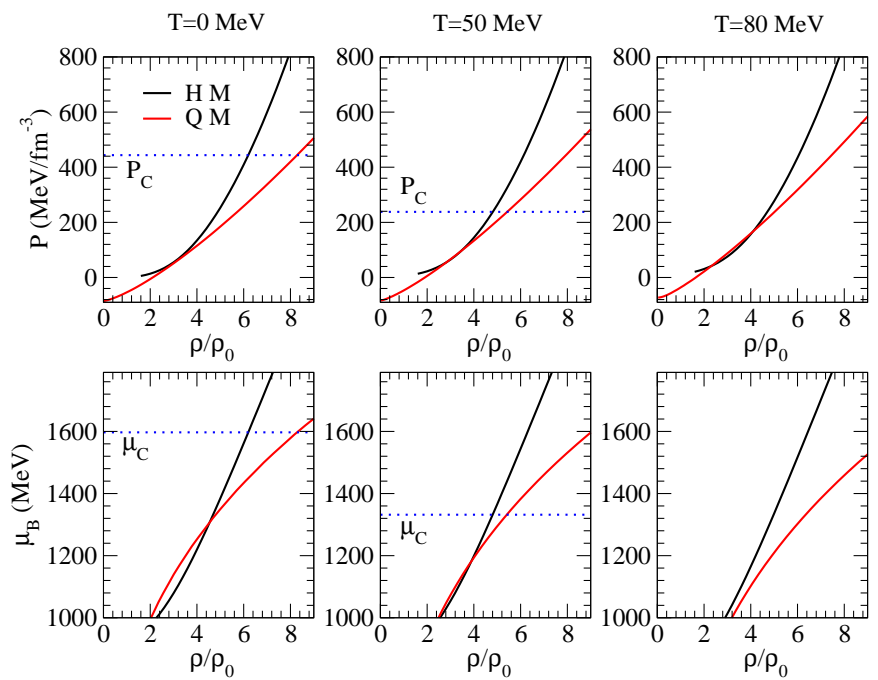

FIG. 4: Maxwell construction for symmetric $(\alpha=0.0)$ matter at temperatures $\mathrm{T}=0,50$ and $80 \mathrm{MeV}$. The dashed lines correspond to the coexistence values of pressure (upper panels) and chemical potential (lower panels). Hadron EoS (black curves) from $N L \rho$ interaction; Quark $E o S$ (grey curves) from $M I T$ bag model with $B^{1 / 4}=160 \mathrm{MeV}$.

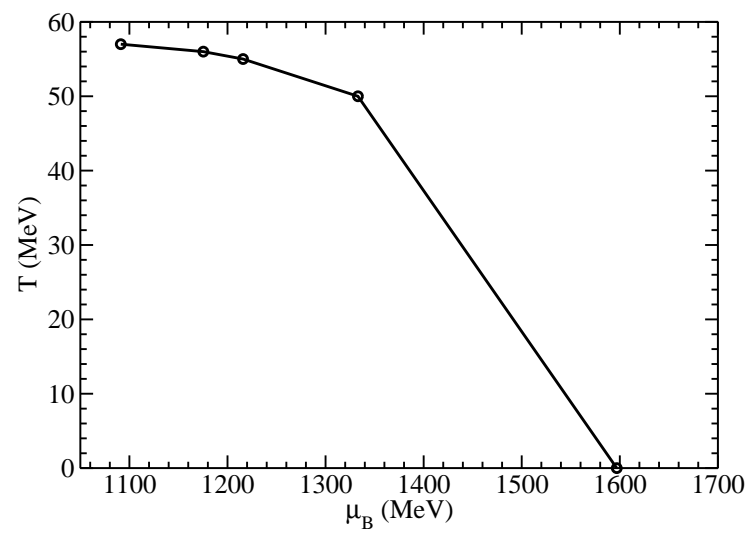

FIG. 5: Phase transition line in the $(T, \mu)$ plane for symmetric $(\alpha=0.0)$ matter. Hadron and Quark EoS like in Fig 4

sults are shown in Fig 4 for the same hadron and quark $E o S$ 's as in Fig 3 at temperatures T=0, 50 and $80 \mathrm{MeV}$. The equal chemical potential densities (intersection of the dotted line in the lower panel) must correspond to the equal pressure densities of the upper panels. We nicely see that at $\mathrm{T}=0 \mathrm{MeV}$ the mixed phase is centered around $\rho / \rho_{0} \simeq 7.0$, exactly the $\alpha=0$ crossing point of Fig,1, confirming our energetic argument about the transition location. Precisely the two boundaries are at $\rho_{H} / \rho_{0}=6.2$ and $\rho_{Q} / \rho_{0}=8.3$ at a chemical potential $\mu=1597.0 \mathrm{MeV}$. 


\section{About the Critical End Point}

From Fig 4 we also see that the size of the mixed phase is shrinking with temperature, it is very narrow at $\mathrm{T}=50$ $\mathrm{MeV}$ and finally at $\mathrm{T}=80 \mathrm{MeV}$ we cannot have anymore a first order transition. In fact a kind of Critical End Point is appearing at $T_{c} \simeq 58 \mathrm{MeV}, \rho_{c} / \rho_{0} \simeq 3.8, P_{c} \simeq$ $120 \mathrm{MeV} / \mathrm{fm}^{3}$ and $\mu_{c} \simeq 1090 \mathrm{MeV}$, see also Fig 3, The result is dependent on the choice of the Bag constant, with an increase of the critical temperature with the Bag value due to the reduction of the pressure in the quark phase, while the chemical potentials are not affected.

However, as already noted in the introduction, within the present Two-EoS approach it is impossible to discuss the nature of the transition around this apparent Critical End Point. The fact that we reach a point with equal densities in the two phases is not implying the onset of a continous transition. Indeed from the coexistence conditions of a first order we can have a point with equal densities but with still a gap in the entropy densities. Since we can follow the transition in the $(T, \mu)$ plane, such point will correspond to a zero of the $d T / d \mu$, from the Clausius-Clapeyron Equation.

We have checked this possibility for the transition discussed before, see Fig 4] of symmetric matter. In Fig [5 we present the calculated points of the phase diagram in the $(T, \mu)$ plane. We see that approaching the end point of the binodal surface we come very close to the $d T / d \mu=0$ condition and so we cannot deduce that we have reached a real Critical End Point of the first order transition.

We note that this result is not implying that the properties of the mixed phase at lower temperatures discussed within Two-EoS models are meaningless. We can trust them if we are using "realistic" effective interactions in the two phases. In fact this is the main point raised here, where the focus is on the isospin dependence of the mixed phase at low temperature, that can be probed in heavy ion collisions at intermediate energies.

\section{INSIDE THE MIXED PHASE OF ASYMMETRIC MATTER}

For $\alpha=0.2$ asymmetric matter, in the Figs 6 , 7 we show also the $\left(T, \rho_{B}\right)$ curves inside the Mixed Phase corresponding to a $20 \%$ and $50 \%$ presence of the quark component $(\chi=0.2,0.5)$, evaluated respectively with the two choices, $N L \rho$ and $N L \rho \delta$, of the symmetry interaction in the hadron sector. We note, as also expected from Fig 2 , that in the more repulsive $N L \rho \delta$ case the lower boundary is much shifted to the left. However this effect is not so evident for the curve corresponding to a $20 \%$ quark concentration, and almost absent for the $50 \%$ case. The conclusion seems to be that for a stiffer symmetry term in a heavy-ion collision at intermediate energies during the compression stage we can have more chance to probe the mixed phase, although in a region with small weight

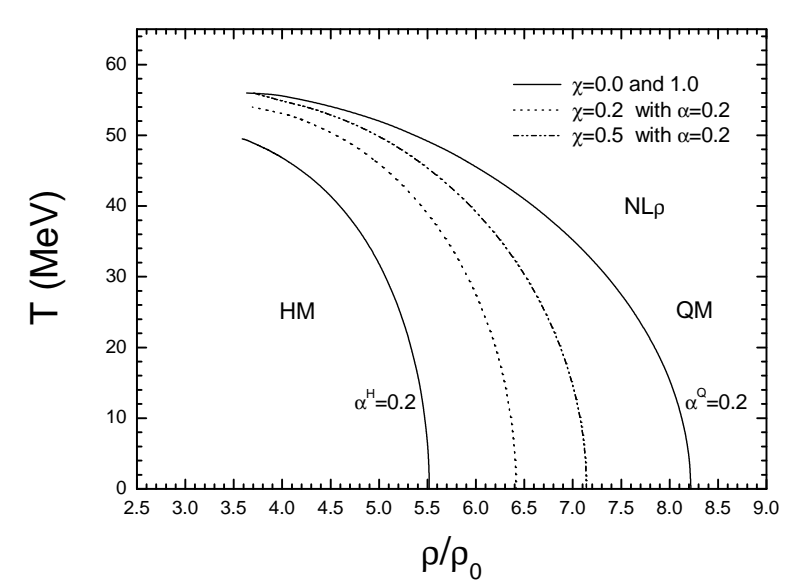

FIG. 6: Asymmetric $\alpha=0.2$ matter. Binodal surface and $\left(T, \rho_{B}\right)$ curves for various quark concentrations $(\chi=0.2,0.5)$ in the mixed phase. Quark EoS: MIT Bag model with $B^{1 / 4}=160 \mathrm{MeV}$. Hadron $\operatorname{EoS}: N L \rho$ Effective Interaction.

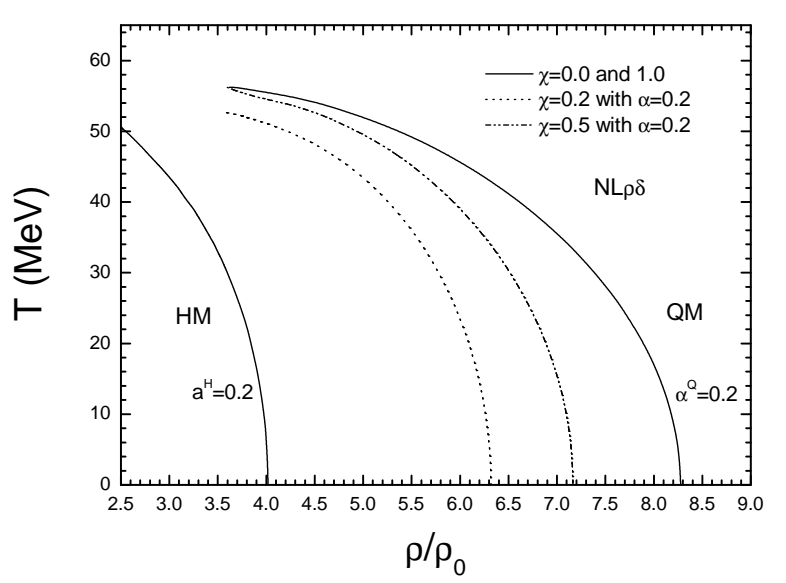

FIG. 7: As in Fig 6 for the $N L \rho \delta$ Effective Interaction in the Hadron sector.

of the quark component.

In fact from the solution of the system Eq.(4) we get the baryon densities $\rho_{B}^{H}, \rho_{B}^{Q}$ in the two phases for any $\chi$ value. In the Figs. 8, 9 we present the results for the same weights $20 \%, 50 \%$ of the quark phase of the previous figures. The quark phase appears always with larger baryon density, even for the lowest value of the concentration.

Can we expect some signatures related to the subsequent hadronization in the following expansion?

An interesting possibility is coming from the study of 


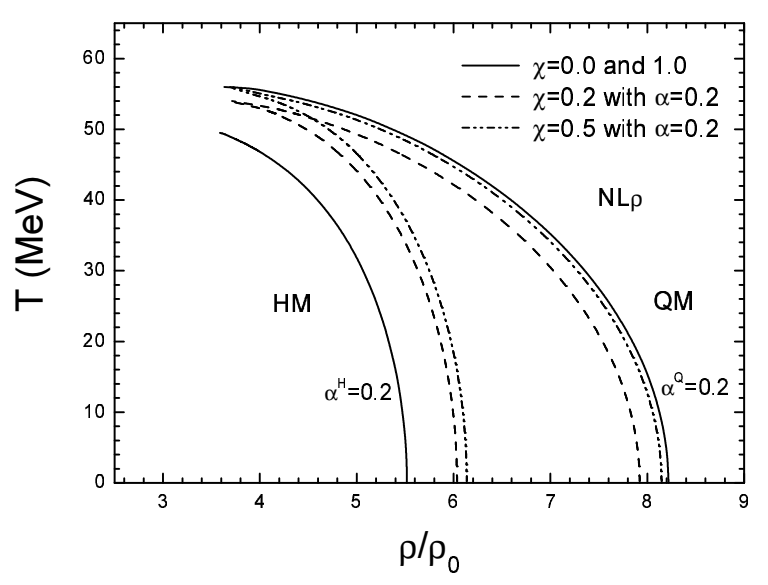

FIG. 8: Asymmetric $\alpha=0.2$ matter. Binodal surface and $\left(T, \rho_{B}^{H}, \rho_{B}^{Q}\right)$ curves for various quark concentrations in the mixed phase. Quark EoS: MIT Bag model with $B^{1 / 4}=160$ $M e V$. Hadron EoS: NL $\rho$ Effective Interaction.

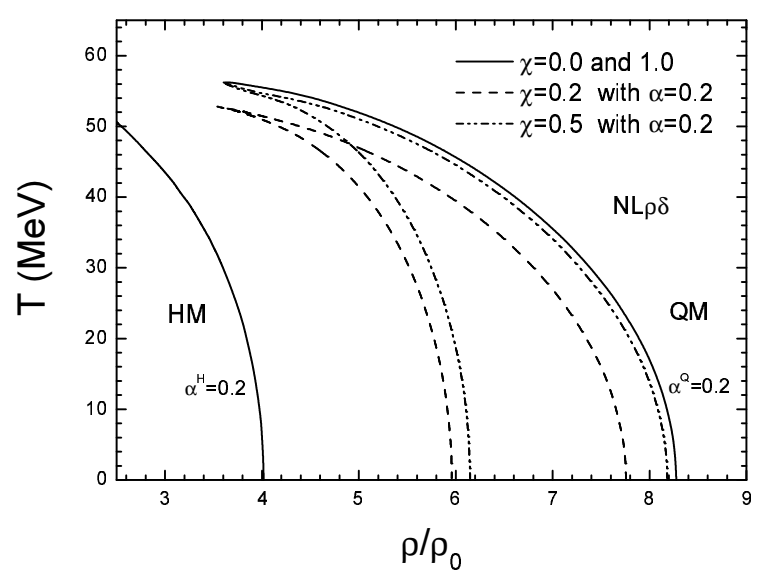

FIG. 9: As in Fig 8 , for the $N L \rho \delta$ Effective Interaction in the Hadron sector.

the asymmetry $\alpha^{Q}$ in the quark phase. In fact since the symmetry energy is rather different in the two phases we can expect an Isospin Distillation (or Fractionation), very similar to the one observed in the Liquid-Gas transition in dilute nuclear matter [12, 34, 35], this time with the larger isospin content in the higher density quark phase.

In Fig 10 we show the asymmetry $\alpha^{Q}$ in the quark phase as a function of the quark concentration $\chi$ for the case with global asymmetry $\alpha=0.2$ (zero temperature). The calculation is performed with the two choices of the symmetry term in the hadron sector. We see an impres-

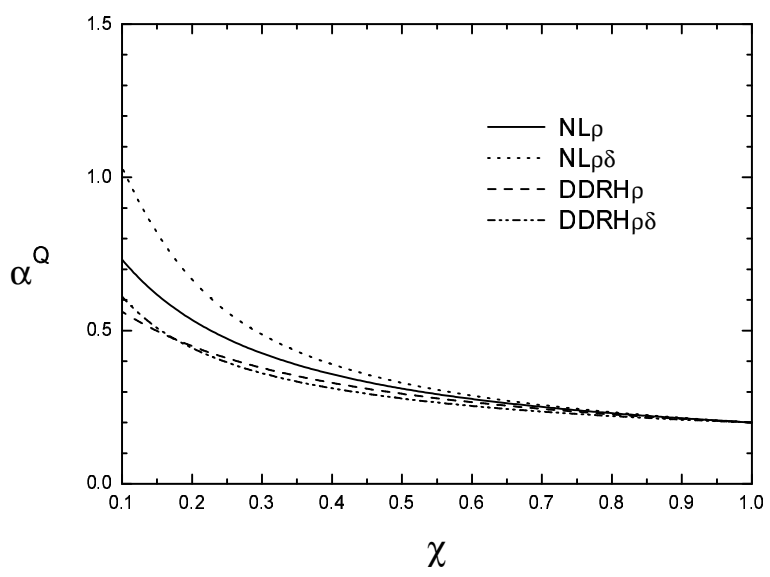

FIG. 10: Quark asymmetry in the mixed phase vs. the quark concentration for asymmetric matter with $T=0$ and $\alpha=0.2$. $N L \rho$ and $N L \rho \delta$ Effective Hadron Interactions are considered. The corresponding results with Density Dependent couplings are also shown, see the Subsection. Quark EoS: MIT bag model with $B^{1 / 4}=160 \mathrm{MeV}$.

sive increase of the quark asymmetry when we approach the lower boundary of the mixed phase, even to values larger than one, likely just for numerical accuracy [36]. Of course the quark asymmetry recovers the global value 0.2 at the upper boundary $\chi=1$. A simple algebraic calculation allows to evaluate the corresponding asymmetries of the hadron phase. In fact from the charge conservation we have that for any $\chi$-mixture the global asymmetry $\alpha$ is given by:

$$
\alpha \equiv-\frac{\rho_{3}}{\rho_{B}}=\frac{(1-\chi) \alpha^{H}}{(1-\chi)+\chi \frac{\rho_{B}^{Q}}{\rho_{B}^{H}}}+\frac{\chi \alpha^{Q}}{(1-\chi) \frac{\rho_{B}^{H}}{\rho_{B}^{Q}}+\chi}
$$

For any $\chi$, from the calculated $\alpha^{Q}$ of Fig 10 and the $\rho_{B}^{H}, \rho_{B}^{Q}$ of Figs 8 , 9, we can get the correspondent asymmetry of the hadron phase $\alpha^{H}$. For a $20 \%$ quark concentration we have an $\alpha^{Q} / \alpha^{H}$ ratio around 5 for $N L \rho$ and around 20 for $N L \rho \delta$, more repulsive in the isovector channel. It is also interesting to compare the isospin content $N / Z$ of the high density region expected from transport simulations without the Hadron-Quark transition and the effective $N / Z$ of the quark phase in a $20 \%$ concentration. In the case of $A u+A u$ (initial $N / Z=1.5$ ) central collisions at $1 \mathrm{AGeV}$ in pure hadronic simulations we get in the high density phase a reduced $N / Z \sim 1.2-1.25$ (respectively with $N L \rho \delta-N L \rho$ interactions) due to the fast neutron emission [30, 31]. The corresponding isospin content of the quark phases is much larger, $N / Z=3.0$ for $N L \rho$ and $N / Z=5.7$ for $N L \rho \delta$. This is the neutron trapping effect discussed in the Section 5. We would expect a signal of such large asym- 


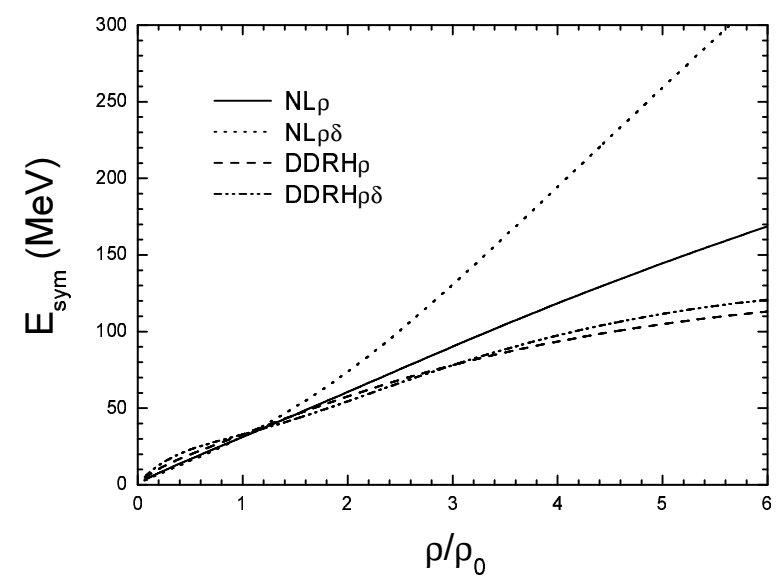

FIG. 11: Density dependence of the symmetry energy for the used RMF hadron effective models. $N L \rho$ and $N L \rho \delta$ represent the Non-Linear Effective Hadron Interactions with constant couplings. The corresponding results with Density Dependent couplings are also shown.

metries, coupled to a larger baryon density in the quark phase, in the subsequent hadronization.

We finally remark that at higher temperature and smaller baryon chemical potential (ultrarelativistic collisions) the isospin effects discussed here are going to vanish [37], even if other physics can enter the game and charge asymmetry effects are predicted also at $\mu_{B}=0$ and $T \simeq 170 \mathrm{MeV}$ [38, 39].

\section{Results with a softer symmetry term at high baryon densities}

In order to account for the the present uncertainties on our knowledge of the symmetry term of the hadron EoS at high baryon density, see also the recent [16], we have performed a new calculation using a RMF hadron interaction which gives a much softer behavior of the symmetry energy at high densities. In this way we can check the "robustness" of the expected isospin effects on the mixed phase discussed before. We use a Density Dependent Relativistic Hadron (DDRH) field approach, where an explicit density dependence of the meson-nucleon couplings is introduced 40 42], see details in Appendix A2. As clearly shown in Fig 11, the main difference with respect to the previously presented results is that the symmetry energy is now less repulsive at high density. This is due to the fact that, following some indications from DiracBrueckner calculations [43, 44], the isovector-meson couplings in the $D D R H \rho$ and $D D R H \rho \delta$ cases show an increase for the attractive $\delta$-field and a decrease for the repulsive $\rho$-field, see Fig 14 in Appendix A2.

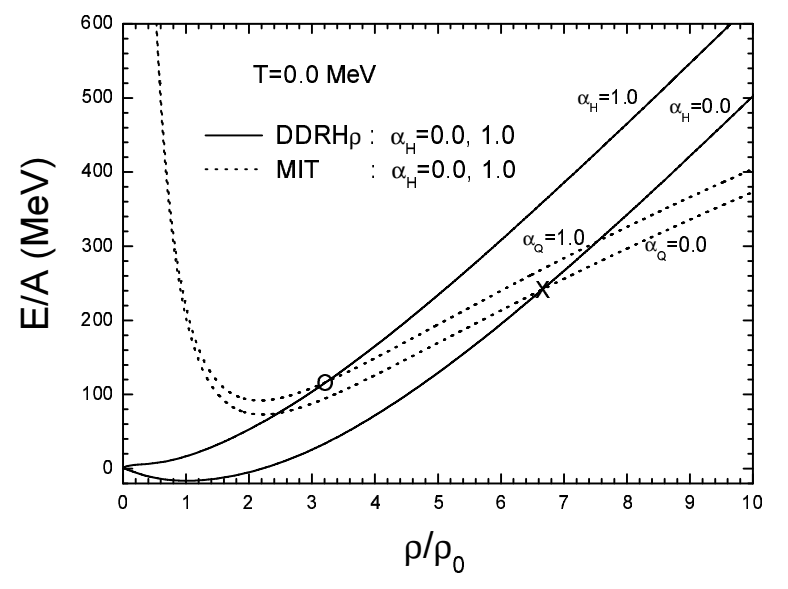

FIG. 12: Zero temperature EoS of Symmetric/Neutron Matter: Hadron $(D D R H \rho)$, solid lines, vs. Quark (MIT-Bag), dashed lines. $\alpha_{H, Q}$ represent the isospin asymmetry parameters respectively of the hadron,quark matter: $\alpha_{H, Q}=0$, Symmetric Matter; $\alpha_{H, Q}=1$, Neutron Matter.

Moreover interesting rearrangement terms are now present in the pressure and in the baryon chemical potentials, proportional to the density slopes of the couplings (see Appendix A2) and so particularly important at high densities, as also shown in neutron star applications [45].

We present first some results on the shift to lower densities of the onset of the mixed phase with increasing isospin asymmetry. In Fig 12 we have the result with $D D R H \rho$ supporting the crossing argument of the Fig[1. Fig 13 shows in more detail the shift to the left of the lower boundaries. The curves should be compared to the corresponding lines of the $N L$-constant coupling model: $D D R H$ to the solid lines of Fig $3(N L, \alpha=0.0)$, $D D R H \rho$ to the solid lines of Fig $[(N L \rho, \alpha=0.2)$ and finally $D D R H \rho \delta$ to the solid lines of Fig $7(N L \rho \delta$, $\alpha=0.2)$. We see that the isospin effects of the hadronquark transition are still present, although with some reduction.

Finally the new Isospin Distillation effects are shown as the $D D R H$ results added in Fig 10, about the isospin asymmetry in the quark phase for different quark concentrations. We note that for $20 \%-30 \%$ quark components we still see a noticeable increase of the isospin asymmetry.

We can conclude that the revealed isospin asymmetry effects on the hadron-quark mixed phase at high baryon density appear to be rather "robust" with respect to relatively large variations of the stiffness of the symmetry term. 


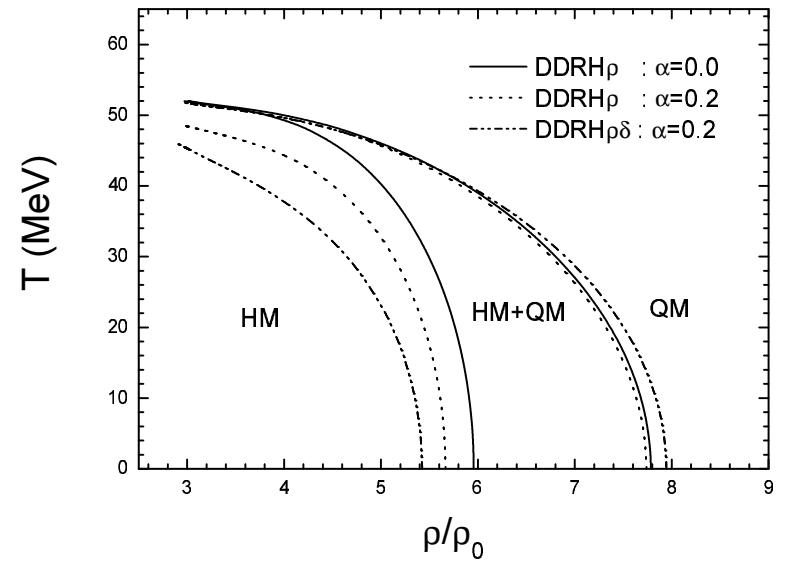

FIG. 13: Binodal surface for symmetric $(\alpha=0.0)$ and asymmetric $(\alpha=0.2)$ matter. Hadron EoS from $D D R H$ interactions. Quark EoS: $M I T$ bag model with $B^{1 / 4}=160 \mathrm{MeV}$.

\section{ISOSPIN IN EFFECTIVE QUARK MODELS}

All the above results will be also sensitive to the explicit inclusion of isovector interactions in effective nonperturbative QCD models at high baryon chemical potentials. Unfortunately few attempts have been worked out for two main reasons: i) the difficulties of latticeQCD calculations at high baryon densities; ii) the main interest on the QGP phase transition at high temperature and small baryon chemical potentials, as probed in the expanding fireball of ultrarelativistic heavy ion collisions. A first approach can be supplied by a two-flavor Nambu-Jona Lasinio $(N J L)$ model [19], which in fact describes the chiral restoration but not the deconfinement dynamics. The isospin asymmetry can be included in a flavor-mixing picture [20, 46], corresponding to different couplings to the $(\mathrm{u}, \mathrm{d})$ quark-antiquark condensates. As a consequence we can have now a dependence of the constituent mass of a given flavor to both quark condensates. We devote the Appendix B to a detailed study of this isospin effects in the $N J L$ chiral dynamics.

Due to the scalar nature of the interacting part of the corresponding Lagrangians only the quark effective mass dynamics will be affected. In the "realistic" small mixing case, see also [46, 47], we get a definite $M_{u}^{*}>M_{d}^{*}$ splitting at high baryon density (before the chiral restoration).

All that can indicate a more fundamental confirmation of the $m_{p}^{*}>m_{n}^{*}$ splitting in the hadron phase, as suggested by the effective $Q H D$ model with the isovector scalar $\delta$ coupling, see [12, 23]. However such isospin mixing effect results in a very small variation of the symmetry energy in the quark phase, related only to the Fermi kinetic contribution. Moreover we remind that confine- ment is still missing in this $N J L$ mean field approach. In any case there are extensive suggestions about a favored chiral symmetry restoration in systems with large neutron excess [49].

More generally starting from the QCD Lagrangian one can arrive to an effective color current-current interaction where an expansion in various components can provide isovector contributions, [50].

In this respect we remark another interesting "indirect" isospin effect, i.e. not directly coming from isovector terms in the effective Lagrangian, but related to the presence of quark condensates due to the attractive gluon interaction. We note that just a color-pairing mechanism in the two-flavor system (the $2 S C$ phase [51]) would imply a stiffer symmetry energy in the quark EoS since we have a larger attraction when the densities of up and down quarks are equal. A first study of the high density hadron-quark transition including such gluon correlation in the Bag model has been presented very recently in [21]. Now the symmetry energy difference between hadron and quark phases is partially reduced, at least at low temperatures, and consequently also the isospin effects discussed in detail in this work will be weaker, although still present. An interesting point is that in any case the quark phase is more bound due to the attractive gluon contribution. Hence the transition to the mixed phase will still appear at relatively low baryon densities, now for an "isoscalar" mechanism, within the reach of "low energy" heavy ion collisions, i.e. in the range of few $\mathrm{AGeV}$. As an intuitive picture we can refer again to the Fig.1. Essentially the difference between the $\alpha_{Q}=0.0$ and $\alpha_{Q}=1$ curves is increasing but meanwhile both are decreasing.

With increasing temperature the color pairing effect will be in general reduced, as confirmed in 52 in an extended $N J L$ calculation, and so isospin effects, as discussed before, will be more relevant. All that is naturally related to the used value of the superconducting gap, opening new stimulating perspectives. In this sense new experiments on mixed phase properties observed with isospin asymmetric heavy ion collisions, as suggested in the final section, will be extremely important.

\section{PERSPECTIVES AND SUGGESTED OBSERVABLES}

Based on the qualitative argument of the Introduction and on more detailed calculations in a first order phase transition scheme, we have predicted rather "robust" isospin effects on the hadron-quark transition at high baryon densities, not depending on details of the EoS parametrizations in the hadron and quark phases.

Our results seem to indicate a specific region where the onset of the mixed phase should be mainly located: $2<$ $\rho_{B} / \rho_{0}<4, T \leq 50-60 \mathrm{MeV}$, for realistic asymmetries $\alpha \sim 0.2-0.3$. A key question is if such a region of the phase space can be explored by means of Heavy-Ion- 
Collisions. In refs. [2, 3] it is shown that even collisions of stable nuclei at intermediate energies $(E / A \sim 1-2 \mathrm{GeV})$ make available the pertinent $\left(T, \rho_{B}, \alpha\right)$ region where the phase transition is expected to occur.

In this respect we can refer to the reaction ${ }^{238} U+{ }^{238} U$ (average isospin asymmetry $\alpha=0.22$ ) at $1 \mathrm{AGeV}$ that has been investigated in ref. [2], using a consistent Relativistic Mean Field approach with the same interactions, for a semicentral impact parameter $b=7 \mathrm{fm}$, chosen just to increase the neutron excess in the interacting region. The evolution of momentum distribution and baryon/isospin densities in a space cell located in the c.m. of the system has been also studied. After about $10 \mathrm{fm} / \mathrm{c}$ a local equilibration is achieved still in the compressed phase, before the fast expansion. We have a unique Fermi distribution and from a simple fit the "local" temperature can be evaluated. A rather exotic nuclear matter is formed in a transient time of the order of $10-20 \mathrm{fm} / \mathrm{c}$, with baryon density around $3-4 \rho_{0}$, temperature $50-60 \mathrm{MeV}$, and isospin asymmetry between 0.2 and 0.3 , likely inside the estimated mixed phase region.

Of course a relatively higher beam energy will allow to enter more deeply into the mixed phase. Such energies will however be available in the next future. In particular we notice that high intensity ${ }^{238} U$ beams in this energy range would be delivered in the first stage of the FAIR facility [4, 53] and also at JINR-Dubna in the Nuclotron first step of the NICA project [37].

Which are the observable effects to look at if we enter and/or cross the mixed phase?

As already stressed, a first expectation will be the Isospin Distillation effect, a kind of neutron trapping in the quark phase, supported by statistical fluctuations [2] as well as by a symmetry energy difference in the two phases, as discussed in Section III. In fact while in the pure hadron matter (neutron-rich) at high density we have a large neutron potential repulsion (in both $N L \rho$, $N L \rho \delta$ as well as in the corresponding $D D R H$ cases), in the quark phase the $d$-quarks see a smaller symmetry repulsion essentially only due to the kinetic contribution from the Fermi gas. As a consequence while in a pure hadronic phase neutrons are quickly emitted or "transformed" in protons by inelastic collisions [31], when the mixed phase starts forming, neutrons are kept in the interacting system, in the quark phase, where they can even thermalize, up to the subsequent hadronization in the expansion stage [3]. Observables related to such neutron "trapping" could be

- (i). An inversion in the trend of emission of fast neutron rich clusters with increasing beam energy, to be seen in the $n / p,{ }^{3} \mathrm{H} /{ }^{3} \mathrm{He}$.. ratios at high kinetic energies;

- (ii). An enhancement of the production of isospinrich nucleon resonances and subsequent decays, that can be evaluated via equilibrium statistical approaches [54];
- (iii). Related to the previous point, an increase of $\pi^{-} / \pi^{+}, K^{0} / K^{+}$yield ratios for mesons coming from high density regions, to be selected via large transverse momenta, corresponding to a large radial flow.

If such kinetic selection of particles from the mixed phase can really be successful also other potential signatures would become available. One is related to the general softening of the matter, due to the contribution of more degrees of freedom, that should show up in the damping of collective flows 55 .

The azimuthal distributions (elliptic flows) will be particularly affected since particles mostly retain their high transverse momenta escaping along directions orthogonal to the reaction plane without suffering much rescattering processes. Thus a further signature could be the observation, for the selected particles, of the onset of a quark-number scaling of the elliptic flow: a property of hadronization by quark coalescence that has been predicted and observed at RHIC energies, i.e. for the transition at $\mu_{B}=0$ [56].

We note that all the above results, on the Binodal Boundaries of the mixed phase and on the Isospin Distillation are sensitive to the symmetry term in the hadron sector, although the main isospin effects are present for all the parametrizations of the isovector interaction. At variance, for the quark sector the lack of explicit isovector terms could strongly affect the location of the phase transition in asymmetric matter and the related expected observables.

In conclusion the aim of this work is twofold:

- To stimulate new experiments on isospin effects in heavy ion collisions at intermediate energies (in a few $A G e V$ range) with attention to the isospin content of produced particles and to elliptic flow properties, in particular for high- $p_{t}$ selections.

- To stimulate more refined models of effective Lagrangians for non-perturbative QCD, where isovector channels are consistently accounted for and/or gluon correlations, leading to diquark condensates, can induce symmetry energy effects.

\section{Acknowledgments}

This project is supported by the National Natural Science Foundation of China under Grant Nos. 10875160, 11075037 and the INFN of Italy. V.B. is grateful for the warm hospitality at Laboratori Nazionali del Sud, INFN. This collaboration is supported in part by the Romanian Ministry for Education and Research under the CNCSIS contract PNII ID-946/2007. 


\section{Appendix A: Equation of state for hadronic matter}

\section{A1. Nonlinear (NL) relativistic mean field model with constant couplings}

A Lagrangian density with "minimal" meson channels and non-linear terms is used. The nuclear interaction is mediated by two isoscalar, the scalar $\sigma$ and the vector $\omega$, and two isovector, the scalar $\delta$ and the vector $\rho$, mesons. Non linear terms are considered only for the $\sigma$ contribution to account for the correct compressibility around saturation. Constant nucleon-meson couplings are used, chosen to reproduce the saturation properties and to represent a reasonable average of the density dependence predicted by Relativistic Dirac-Brueckner-Hartree-Fock (DBHF) calculations [43, 44], see details in refs. [12, 23].

$$
\begin{aligned}
L= & \bar{\psi}\left[i \gamma_{\mu} \partial^{\mu}-\left(M-g_{\sigma} \sigma-g_{\delta} \vec{\tau} \cdot \vec{\delta}\right)\right. \\
& \left.-g_{\omega} \gamma_{\mu} \omega^{\mu}-g_{\rho} \gamma^{\mu} \vec{\tau} \cdot \vec{b}_{\mu}\right] \psi \\
& +\frac{1}{2}\left(\partial_{\mu} \sigma \partial^{\mu} \sigma-m_{\sigma}^{2} \sigma^{2}\right)-U(\sigma) \\
& +\frac{1}{2} m_{\omega}^{2} \omega_{\mu} \omega^{\mu}+\frac{1}{2} m_{\rho}^{2} \vec{b}_{\mu} \cdot \vec{b}^{\mu} \\
& +\frac{1}{2}\left(\partial_{\mu} \vec{\delta} \cdot \partial^{\mu} \vec{\delta}-m_{\delta}^{2} \overrightarrow{\delta^{2}}\right) \\
& -\frac{1}{4} F_{\mu \nu} F^{\mu \nu}-\frac{1}{4} \vec{G}_{\mu \nu} \vec{G}^{\mu \nu}
\end{aligned}
$$

where $F_{\mu \nu} \equiv \partial_{\mu} \omega_{\nu}-\partial_{\nu} \omega_{\mu}, \vec{G}_{\mu \nu} \equiv \partial_{\mu} \vec{b}_{\nu}-\partial_{\nu} \vec{b}_{\mu}$, and the $U(\sigma)$ is the nonlinear potential of $\sigma$ meson : $U(\sigma)=$ $\frac{1}{3} a \sigma^{3}+\frac{1}{4} b \sigma^{4}$.

The EoS for nuclear matter at finite temperature in the mean-field approximation (RMF) is given by the energy density

$$
\begin{aligned}
& \epsilon=2 \sum_{i=n, p} \int \frac{\mathrm{d}^{3} k}{(2 \pi)^{3}} E_{i}^{*}(k)\left(f_{i}(k)+\bar{f}_{i}(k)\right) \\
& +\frac{1}{2} m_{\sigma}^{2} \sigma^{2}+U(\sigma) \\
& +\frac{1}{2} \frac{g_{\omega}^{2}}{m_{\omega}^{2}} \rho_{B}^{2}+\frac{1}{2} \frac{g_{\rho}^{2}}{m_{\rho}^{2}} \rho_{3}^{2}+\frac{1}{2} \frac{g_{\delta}^{2}}{m_{\delta}^{2}} \rho_{s 3}^{2},
\end{aligned}
$$

and pressure

$$
\begin{aligned}
& p=\frac{2}{3} \sum_{i=n, p} \int \frac{\mathrm{d}^{3} k}{(2 \pi)^{3}} \frac{k^{2}}{E_{i}^{*}(k)}\left(f_{i}(k)+\bar{f}_{i}(k)\right) \\
& -\frac{1}{2} m_{\sigma}^{2} \phi^{2}-U(\phi) \\
& +\frac{1}{2} \frac{g_{\omega}^{2}}{m_{\omega}^{2}} \rho_{B}^{2}+\frac{1}{2} \frac{g_{\rho}^{2}}{m_{\rho}^{2}} \rho_{3}^{2}+\frac{1}{2} \frac{g_{\delta}^{2}}{m_{\delta}^{2}} \rho_{s 3}^{2}
\end{aligned}
$$

where $E_{i}^{*}=\sqrt{k^{2}+M_{i}^{* 2}}$. The nucleon effective masses are defined as

$$
\left.M_{i}^{*}=M-g_{\sigma} \sigma \mp g_{\delta} \delta_{3} \quad \text { (- proton },+ \text { neutron }\right) \text {. }
$$

The field equations in the relativistic mean field (RMF) approach are

$$
\begin{aligned}
\sigma & =-\frac{a}{m_{\sigma}^{2}} \sigma^{2}-\frac{b}{m_{\sigma}^{2}} \sigma^{3}+\frac{g_{\sigma}}{m_{\sigma}^{2}}\left(\rho_{s p}+\rho_{s n}\right) \\
\omega_{0} & =\frac{g_{\omega}}{m_{\omega}^{2}} \rho \\
b_{0} & =\frac{g_{\rho}}{m_{\rho}^{2}} \rho_{3} \\
\delta_{3} & =\frac{g_{\delta}}{m_{\delta}^{2}}\left(\rho_{s p}-\rho_{s n}\right)
\end{aligned}
$$

with the baryon density $\rho \equiv \rho_{B}^{H}=\rho_{p}+\rho_{n}$ and $\rho_{3}^{H}=\rho_{p}-\rho_{n}, \rho_{s p}$ and $\rho_{s n}$ are the scalar densities for proton and neutron, respectively. The $f_{i}(k)$ and $\bar{f}_{i}(k)$ in Eqs. A2A3 are the fermion and antifermion distribution functions for protons and neutrons $(i=p, n)$ :

$$
\begin{aligned}
f_{i}(k) & =\frac{1}{1+\exp \left\{\left(E_{i}^{*}(k)-\mu_{i}^{*}\right) / T\right\}}, \\
\bar{f}_{i}(k) & =\frac{1}{1+\exp \left\{\left(E_{i}^{*}(k)+\mu_{i}^{*}\right) / T\right\}} .
\end{aligned}
$$

where the effective chemical potentials $\mu_{i}^{*}$ is determined by the nucleon densities $\rho_{i}$

$$
\rho_{i}=2 \int \frac{\mathrm{d}^{3} k}{(2 \pi)^{3}}\left(f_{i}(k)-\bar{f}_{i}(k)\right),
$$

while the scalar densities $\rho_{s, i}$, which gives the coupling to the scalar fields are given by

$$
\rho_{s, i}=2 \int \frac{\mathrm{d}^{3} k}{(2 \pi)^{3}} \frac{M_{i}^{*}}{E_{i}^{*}}\left(f_{i}(k)+\bar{f}_{i}(k)\right),
$$

note the $M_{i}^{*} / E_{i}^{*}$ quenching factor at high baryon density. Clearly at zero temperature the $\mu_{i}^{*}$ reduce to the in medium Fermi energies $E_{F i} *=\sqrt{k_{F i}^{2}+M_{i}^{* 2}}$.

The $\mu_{i}^{*}$ are related to the chemical potentials $\mu_{i}=$ $\partial \epsilon / \partial \rho_{i}$ in terms of the vector meson mean fields by the equation

$$
\begin{aligned}
\mu_{i} & =\mu_{i}^{*}+\frac{g_{\omega}^{2}}{m_{\omega}^{2}} \rho \mp \frac{g_{\rho}^{2}}{m_{\rho}^{2}} \rho_{3} \\
(i & =n, p:- \text { neutron },+ \text { proton })
\end{aligned}
$$

The baryon and isospin chemical potentials in the hadron phase can be expressed in terms of the $(p, n)$ ones as

$$
\mu_{B}^{H}=\frac{\mu_{p}+\mu_{n}}{2}, \quad \mu_{3}^{H}=\frac{\mu_{p}-\mu_{n}}{2} .
$$

In presence of the coupling to the two isovector $\rho, \delta$ meson fields, the expression for the symmetry energy has a simple transparent form, see [12, 23, 57]: 
Table 2. Saturation properties of nuclear matter.

$$
E_{\text {sym }}(\rho)=\frac{1}{6} \frac{k_{F}^{2}}{E_{F}^{*}}+\frac{1}{2}\left[f_{\rho}-f_{\delta}\left(\frac{M^{*}}{E_{F}^{*}}\right)^{2}\right] \rho,
$$

where $M^{*}=M-g_{\sigma} \sigma$ and $E_{F}{ }^{*}=\sqrt{k_{F}^{2}+M^{* 2}}$.

Now we easily see that in the $N L \rho \delta$ choice we have a large increase of the symmetry energy at high baryon densities. The potential simmetry term is given by the combination $\left[f_{\rho}-f_{\delta}\left(M^{*} / E_{F}^{*}\right)^{2}\right]$ of the repulsive vector $\rho$ and attractive scalar $\delta$ isovector couplings. Thus, when the $\delta$ is included we have to increase the $\rho$-meson coupling in order to reproduce the same asymmetry parameter $a_{4}$ at saturation. The net effect will be a stiffer symmetry energy at higher baryon densities due to the $M^{*} / E_{F}^{*}$ quenching of the attractive part. Of course this mechanism can be largely modified if some density dependece is explicitly included in the meson-nucleon couplings, as we will see in the $D D R H$ forces (Appendix A2), also used in this paper..

\section{Parameter determination}

The coupling constants are fixed from good saturation properties and from averaged Dirac-Brueckner-HartreeFock estimations, see the detailed discussions in refs. 12, 23, 57]. DBHF indications of a density dependence of the meson-nucleon couplings at high baryon densities will be accounted for in the $D D R H$ forces of the next Subsection A2.

The isoscalar part of the EoS is chosen to be rather soft at high densities, see [58], in order to satisfy the experimental constraints from collective flows and kaon production in intermediate energy heavy ion collisions [25, 26].

The coupling constants, $f_{i} \equiv g_{i}^{2} / m_{i}^{2}, i=\sigma, \omega, \rho, \delta$, and the two parameters of the $\sigma$ self-interacting terms : $A \equiv a / g_{\sigma}^{3}$ and $B \equiv b / g_{\sigma}^{4}$ are reported in Table 1 . The $\sigma$ mass is fixed at $550 \mathrm{MeV}$. The corresponding properties of nuclear matter are listed in Table 2. Here the binding energy is defined $E / A=\epsilon / \rho-M$.

Table 1. Parameter set.

\begin{tabular}{c|c|c}
\hline Parameter Set & $\mathrm{NL} \rho$ & $\mathrm{NL} \rho \delta$ \\
\hline$f_{\sigma}\left(\mathrm{fm}^{2}\right)$ & 10.32924 & 10.32924 \\
\hline$f_{\omega}\left(\mathrm{fm}^{2}\right)$ & 5.42341 & 5.42341 \\
\hline$f_{\rho}\left(\mathrm{fm}^{2}\right)$ & 0.94999 & 3.1500 \\
\hline$f_{\delta}\left(\mathrm{fm}^{2}\right)$ & 0.000 & 2.500 \\
\hline$A\left(\mathrm{fm}^{-1}\right)$ & 0.03302 & 0.03302 \\
\hline$B$ & -0.00483 & -0.00483 \\
\hline
\end{tabular}

\begin{tabular}{|c|c|}
\hline$\rho_{0}\left(f m^{-3}\right)$ & 0.16 \\
\hline$E / A(M e V)$ & -16.0 \\
\hline$K(\mathrm{MeV})$ & 240.0 \\
\hline$E_{\text {sym }}(\mathrm{MeV})$ & 31.3 \\
\hline$M^{*} / M$ & 0.75 \\
\hline
\end{tabular}

We finally note that these Lagrangians have been already used for flow [29], pion production [30], isospin tracer [59] and kaon production [31] calculations for relativistic heavy ion collisions with an overall good agreement to data.

\section{A2. DDRH forces: Relativistic Mean Field model with density dependent couplings}

The "minimal" Lagrangian density has the same form of the Eq. A1 , now with density dependent couplings and of course without non-linear terms (the $U(\sigma)$ potential). Apart the effect of an explicit variation of the meson-nucleon couplings with baryon density we will expect new terms in the variational derivative of the Lagrangian density, the rearrangement terms $\Sigma_{\mu}^{R}$ that will affect the nucleon field equation as well as the energymomentum tensor and so the $E o S$ and the nucleon chemical potentials, see [40, 41, 45]. The nucleon field equation in a mean field approximation $(R M F)$ is

$$
\begin{aligned}
& \left(i \gamma_{\mu} \partial^{\mu}-\left(M-g_{\sigma} \sigma-g_{\delta} \tau_{3} \delta_{3}\right)-g_{\omega} \gamma^{0} \omega_{0}\right. \\
& \left.-g_{\rho} \gamma^{0} \tau_{3} b_{0}+\gamma^{0} \Sigma_{0}^{R}\right) \psi=0
\end{aligned}
$$

with

$$
\begin{aligned}
& \sigma=\frac{g_{\sigma}}{m_{\sigma}^{2}} \rho_{s}=\frac{g_{\sigma}}{m_{\sigma}^{2}}\left(\rho_{s p}+\rho_{s n}\right) \\
& \omega_{0}=\frac{g_{\omega}}{m_{\omega}^{2}}<\bar{\psi} \gamma^{0} \psi>=\frac{g_{\omega}}{m_{\omega}^{2}} \rho=\frac{g_{\omega}}{m_{\omega}^{2}}\left(\rho_{p}+\rho_{n}\right) \\
& b_{0}=\frac{g_{\rho}}{m_{\rho}^{2}}<\bar{\psi} \gamma^{0} \tau_{3} \psi>=\frac{g_{\rho}}{m_{\rho}^{2}} \rho_{3} \\
& \delta_{3}=\frac{g_{\delta}}{m_{\delta}^{2}}<\bar{\psi} \tau_{3} \psi>=\frac{g_{\delta}}{m_{\delta}^{2}} \rho_{s 3}
\end{aligned}
$$

and the rearrangement term

$$
\begin{aligned}
\Sigma_{0}^{R} & =\left(\frac{\partial g_{\sigma}}{\partial \rho}\right) \frac{g_{\sigma}}{m_{\sigma}^{2}} \rho_{s}+\left(\frac{\partial g_{\delta}}{\partial \rho}\right) \frac{g_{\delta}}{m_{\delta}^{2}} \rho_{s 3}^{2} \\
& -\left(\frac{\partial g_{\omega}}{\partial \rho}\right) \frac{g_{\omega}}{m_{\omega}^{2}} \rho^{2}-\left(\frac{\partial g_{\rho}}{\partial \rho}\right) \frac{g_{\rho}}{m_{\rho}^{2}} \rho_{3}^{2},
\end{aligned}
$$

where $\rho_{3}=\rho_{p}-\rho_{n}$ and $\rho_{s 3}=\rho_{s p}-\rho_{s n}$, with $\rho_{i}, \rho_{s i}$ $(\mathrm{i}=\mathrm{n}, \mathrm{p})$ the nucleon and the scalar densities, see Subsection A1.

Neglecting the derivatives of meson fields, the energymomentum tensor in $R M F$ approximation is given by 


$$
\begin{gathered}
T_{\mu \nu}=i \bar{\psi} \gamma_{\mu} \partial_{\nu} \psi+\left[\frac{1}{2} m_{\sigma}^{2} \sigma^{2}-\frac{1}{2} m_{\omega}^{2} \omega_{\lambda} \omega^{\lambda}\right. \\
\left.-\frac{1}{2} m_{\rho}^{2} \overrightarrow{b_{\lambda}} \overrightarrow{b^{\lambda}}+\frac{1}{2} m_{\delta}^{2} \vec{\delta}^{2}+\bar{\psi} \Sigma_{\lambda}^{R} \gamma^{\lambda} \psi\right] g_{\mu \nu} .
\end{gathered}
$$

The equation of state $(E O S)$ for nuclear matter at finite temperature can be obtained from the thermodynamic potential. Using the above meson field equations A17, the energy density has the form

$$
\begin{array}{ll}
\epsilon=\sum_{i=n, p} 2 \int \frac{\mathrm{d}^{3} k}{(2 \pi)^{3}} E_{i}^{*}(k)\left(f_{i}(k)+\bar{f}_{i}(k)\right)+\frac{1}{2} \frac{g_{\sigma}^{2}}{m_{\sigma}^{2}} \rho_{s}^{2} \\
+\frac{1}{2} \frac{g_{\omega}^{2}}{m_{\omega}^{2}} \rho^{2}+\frac{1}{2} \frac{g_{\rho}^{2}}{m_{\rho}^{2}} \rho_{3}^{2}+\frac{1}{2} \frac{g_{\delta}^{2}}{m_{\delta}^{2}} \rho_{s 3}^{2},
\end{array}
$$

and the pressure

$$
\begin{aligned}
& p=\sum_{i=n, p} \frac{2}{3} \int \frac{\mathrm{d}^{3} k}{(2 \pi)^{3}} \frac{k^{2}}{E_{i}^{*}(k)}\left(f_{i}(k)+\bar{f}_{i}(k)\right)-\frac{1}{2} \frac{g_{\sigma}^{2}}{m_{\sigma}^{2}} \rho_{s}^{2} \\
& +\frac{1}{2} \frac{g_{\omega}^{2}}{m_{\omega}^{2}} \rho^{2}+\frac{1}{2} \frac{g_{\rho}^{2}}{m_{\rho}^{2}} \rho_{3}^{2}-\frac{1}{2} \frac{g_{\delta}^{2}}{m_{\delta}^{2}} \rho_{s 3}^{2}-\Sigma_{o}^{R} \rho .
\end{aligned}
$$

The nucleon chemical potentials $\mu_{i}$ are given in terms of the vector meson mean fields as in the constant coupling case, Eq.(A13), apart the new rearrangement term

$$
\begin{gathered}
\mu_{i}=\mu_{i}^{*}+\frac{g_{\omega}^{2}}{m_{\omega}^{2}} \rho \mp \frac{g_{\rho}^{2}}{m_{\rho}^{2}} \rho_{3}-\Sigma_{o}^{R} \\
(i=n, p:- \text { neutron },+ \text { proton })
\end{gathered}
$$

with the effective masses related to the scalar fields as before

$$
\begin{aligned}
M_{i}{ }^{*} & =M-\frac{g_{\sigma}^{2}}{m_{\sigma}^{2}} \rho_{s} \mp \frac{g_{\delta}^{2}}{m_{\delta}^{2}} \rho_{s 3} \\
& (i=n, p:+ \text { neutron },- \text { proton })
\end{aligned}
$$

\section{Density dependence parametrization}

A general form of parametrization for the density dependence of the meson-nucleon couplings can be given by :

$$
g_{i}(\rho)=g_{i}\left(\rho_{0}\right) f_{i}(x), \quad \text { for } \quad i=\sigma, \omega, \rho, \delta,
$$

with $x=\rho / \rho_{0}$ and $\rho_{0}$ saturation density. As already mentioned the $f_{i}(x)$ are chosen in order to reproduce the density dependence of the couplings deduced from microscopic DBHF calculations. For symmetric matter

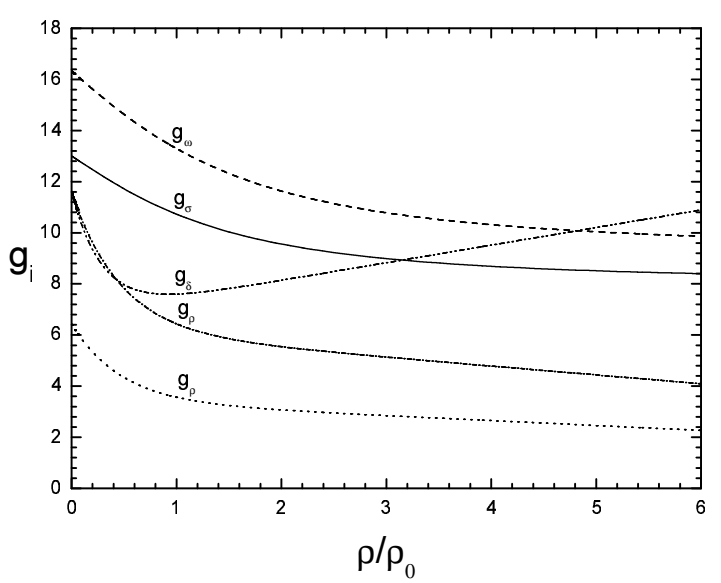

FIG. 14: Density dependence of the meson-nucleon couplings in the used $D D R H$ interactions. The $g_{\rho}$ dotted line at the bottom corresponds to the $D D R H \rho$ case, without the $\delta$ meson.

this analysis has been performed in ref.[41] using for the isoscalar mesons a functional of the form

$$
f_{i}(x)=a_{i} \frac{1+b_{i}\left(x+d_{i}\right)^{2}}{1+c_{i}\left(x+d_{i}\right)^{2}}, \quad \text { for } \quad i=\sigma, \omega,
$$

In the case of asymmetric matter the following parametrization has been proposed for the isovector couplings [42] :

$$
f_{i}(x)=a_{i} \exp \left[-b_{i}(x-1)\right]-c_{i}\left(x-d_{i}\right), \quad \text { for } i=\rho, \delta .
$$

in this way it easier to reproduce the important difference of the $\delta, \rho$ couplings at high density.

We follow the two suggestions, the parametrization form and parameters of our $D D R H$ forces are taken from ref. [41] for $\sigma, \omega$ mesons and ref.[42] for $\rho, \delta$ mesons, respectively. All parameters are listed in Table 3. The density dependent couplings as a function of baryon density are displayed in Fig 14

Table 3. DDRH Parameters.

\begin{tabular}{c|c|c|c|c|c}
\hline Model & \multicolumn{2}{|c|}{$D D R H$} & DDRH $\rho$ & \multicolumn{2}{c}{ DDRH $\rho \delta$} \\
\hline Meson & $\sigma$ & $\omega$ & $\rho$ & $\rho$ & $\delta$ \\
\hline$m_{i}(\mathrm{MeV})$ & 550 & 783 & 770 & 770 & 980 \\
\hline$g_{i}\left(\rho_{0}\right)$ & 10.73 & 13.29 & 3.59 & 6.48 & 7.59 \\
\hline$a_{i}$ & 1.37 & 1.40 & 0.095 & 0.095 & 0.02 \\
\hline$b_{i}$ & 0.23 & 0.17 & 2.17 & 2.17 & 3.47 \\
\hline$c_{i}$ & 0.41 & 0.34 & 0.05 & 0.05 & -0.09 \\
\hline$d_{i}$ & 0.90 & 0.98 & 17.84 & 17.84 & -9.81 \\
\hline
\end{tabular}


The choice of the $g_{i}\left(\rho_{0}\right)$ couplings at saturation is performed in order to have the same nuclear matter normal properties of the Non Linear RMF models (Table 2) of the Subsection A1. The EoS of symmetric matter at high density is also not affected, as we can see comparing the binodal surfaces for zero asymmetry of Figs 3, 13] (solid lines).

At variance, the different behavior of the isovector couplings at high density, increase of $g_{\delta}$ and descrease of $g_{\rho}$, will contribute to get a much softer symmetry energy at high baryon densities, Fig 11. It is easy to check that in this way in nucleonic models of neutron stars the proton fraction limit for the onset of direct URCA processes is hardly reached, see the analysis of ref. 60]. For the purpose of the present paper the use of $D D R H$ interactions is important in order to show that the expected isospin effects on the mixed phase are present even with much softer symmetry terms at high baryon densities.

\section{Appendix B: Nambu-Iona Lasinio model for asymmetric matter}

From the above discussion it appears (extremely) important to include the Isospin degree of freedom in any effective QCD dynamics. A first approach can be supplied by a two-flavor Nambu-Jona Lasinio model where the isospin asymmetry can be included in a flavor-mixing picture [20, 46]. The lagrangian is given by

$$
L=L_{0}+L_{1}+L_{2}
$$

with $L_{0}$ the free part

$$
L_{0}=\bar{\psi}(i \not \partial-m) \psi
$$

and the two different interaction part given by

$$
\begin{aligned}
& L_{1}=G_{1}\left\{(\bar{\psi} \psi)^{2}+(\bar{\psi} \vec{\tau} \psi)^{2}+\left(\bar{\psi} i \gamma_{5} \psi\right)^{2}+\left(\bar{\psi} i \gamma_{5} \vec{\tau} \psi\right)^{2}\right\} \\
& \left.L_{2}=G_{2}\left\{(\bar{\psi} \psi)^{2}-(\bar{\psi} \vec{\tau} \psi)^{2}-\left(\bar{\psi} i \gamma_{5} \psi\right)^{2}+\left(\bar{\psi} i \gamma_{5} \vec{\tau} \psi\right)^{2}\right\} \mathrm{B} 2\right)
\end{aligned}
$$

In the mean field approximation the new Gap Equations are $M_{i}=m_{i}-4 G_{1} \Phi_{i}-4 G_{2} \Phi_{j}, i \neq j,(u, d)$, where the
$\Phi_{u, d}=<\bar{u} u>,<\bar{d} d>$ are the two (negative) condensates which are given by

$$
\Phi_{f}=-2 N_{c} \int \frac{d^{3} p}{(2 \pi)^{3}} \frac{M_{f}}{E_{p, f}}\left\{1-f^{-}\left(T, \mu_{f}\right)-f^{+}\left(T, \mu_{f}\right)\right\} .
$$

and $m_{u, d}=m$ the (equal) current masses.

Introducing explicitily a flavor mixing, i.e. the dependence of the constituent mass of a given flavor to both condensate, via $G_{1}=(1-\beta) G_{0}, G_{2}=\beta G_{0}$ we have the coupled equations

$$
\begin{aligned}
& M_{u}=m-4 G_{0} \Phi_{u}+4 \beta G_{0}\left(\Phi_{u}-\Phi_{d}\right), \\
& M_{d}=m-4 G_{0} \Phi_{u}+4(1-\beta) G_{0}\left(\Phi_{u}-\Phi_{d}\right) .(\mathrm{B} 4)
\end{aligned}
$$

For $\beta=1 / 2$ we have back the usual NJL $\left(M_{u}=M_{d}\right)$, while small/large mixing is for $\beta \Rightarrow 0 / \beta \Rightarrow 1$ respectively. The value of $\beta$ has a consequence on the structure of the phase diagram in the region of low temperatures and high chemical potential. In fact as shown in [20, 46] for $\beta=0$ there are two distinct phase transitions for the up quarks and for the down quarks, but for this value the interaction is symmetric under $U_{A}(1)$ transformations and it is unrealistic. While for $\beta \geq 0.1$ the $U_{A}(1)$ symmetry becomes explicitly broken and there is only a single first order phase transition. Realistic estimations of $\beta$ fitting the physical $\eta$-meson mass give a value of $\beta \approx 0.11$ [46, 47].

In neutron rich matter $\left|\Phi_{d}\right|$ decreases more rapidly due to the larger $\rho_{d}$ and so $\left(\Phi_{u}-\Phi_{d}\right)<0$. In the "realistic" small mixing case we will get a definite $M_{u}>M_{d}$ splitting at high baryon density (before the chiral restoration). This expectation is confirmed by a full calculation of the coupled gap equations with standard parameters 3, 48]. All that can indicate a more fundamental confirmation of the $m_{p}^{*}>m_{n}^{*}$ splitting in the hadron phase, as suggested by the effective $Q H D$ model with the isovector scalar $\delta$ coupling, see [12, 23].

However such isospin mixing effect results in a very small variation of the symmetry energy in the quark phase, still related only to the Fermi kinetic contribution. In fact this represents just a very first step towards a more complete treatment of isovector contributions in effective quark models, of large interest for the discussion of the phase transition at high densities.
[1] H. Müller, Nucl.Phys. A618 349 (1997).

[2] M. Di Toro, A. Drago, T. Gaitanos, V. Greco, A. Lavagno, Nucl.Phys. A775 102 (2006).

[3] M. Di Toro et al. Progr.Part.Nucl.Phys. 62 389-401 (2009).

[4] P. Senger et al., J.Phys.G:Nucl.Part.Phys. 36064037 (2009).

[5] See the Website < http://nica.jinr.ru/ $>$.

[6] G.F. Burgio, M. Baldo, P.K. Sahu, H.-J. Schulze, Phys.Rev. C66 025802 (2002).

[7] M. Bejger, P. Haensel, J.L. Zdumik, Monthly Notices of the Royal Astronomy Society 359699 (2005).
[8] O.E. Nicotra, M. Baldo, G.F. Burgio, H.-J. Schulze, Phys.Rev. D74 123001 (2006).

[9] M.Baldo, G.F. Burgio, P.Castorina, S. Plumari, D. Zappala', Phya.Rev. C66 035804 (2007).

[10] G.F. Burgio, S. Plumari, Phys.Rev. D77 085022 (2008).

[11] A. Rahbi, H.Pais, P.K. Panda, C. Procidencia, Quarkhadron phase transition in a neutron star with strong magnetic fields, arXiv:0909.1114[nucl-th].

[12] V. Baran, M. Colonna, V. Greco, M. Di Toro, Phys.Rep. 410335 (2005)

[13] C. Fuchs, H.H. Wolter, Eur. Phys. J. A30 5 (2006).

[14] B.A. Li, L.W. Chen, C.M. Ko, Phys.Rep. 465113 (2008). 
[15] W. Trautmann, Nucl. Phys. A834 548c (2010) and arXiv:1001.3867[nucl-exp].

[16] V. Giordano, M. Colonna, M. Di Toro, V. Greco, J. Rizzo, Phys.Rev. C81 044611 (2010).

[17] M. Di Toro, V. Baran, M. Colonna, V. Greco, J. Phys.G: Nucl.Part.Phys. 37083101 (2010).

[18] A. Chodos et al., Phys.Rev. D9 3471 (1974).

[19] Y. Nambu and G. Jona-Lasinio, Phys.Rev. 122345 (1961); 124246 (1961).

[20] M. Buballa, Phys. Rep. 407205 (2005)

[21] G. Pagliara, J. Schaffner-Bielich, Phase transition from nuclear matter to color superconducting quark matter: the effects of isospin, arXiv:1003.1017 [nucl-th].

[22] B.D. Serot and J.D. Walecka, Adv. Nucl. Phys. 161 (1985).

[23] B. Liu, V. Greco, V. Baran, M. Colonna, M. Di Toro, Phys.Rev. C65 045201 (2002).

[24] D. Page, S. Reddy, Ann.Rev.Nucl.Part.Sci. 56327 (2006).

[25] P. Danielewicz, R. Lacey, W.G. Lynch, Science 2981592 (2002).

[26] C. Fuchs, Prog.Part.Nucl.Phys. 56 1-103 (2006).

[27] B. Müller, Rep.Prog.Phys. 58611 (1995).

[28] L.D. Landau and L. Lifshitz, Statistical Physics Pergamon Press 1969, Oxford.

[29] V. Greco et al., Phys.Lett. B562 215 (2003).

[30] T. Gaitanos et al., Nucl.Phys. A732 24 (2004).

[31] G. Ferini, T. Gaitanos, M. Colonna, M. Di Toro. H.H. Wolter, Phys.Rev.Lett. 97202301 (2006).

[32] N.K. Glendenning, S.A. Moszkowski, Phys.Rev.Lett. 67 2414 (1991).

[33] D. Vretenar, T. Niksic, P. Ring, Phys.Rev. C68 024310 (2003), and refs. therein.

[34] V. Baran, M. Colonna, M. Di Toro, A. Larionov, Nucl.Phys. A632 287-303 (1998).

[35] P. Chomaz, M. Colonna, J. Randrup. Phys.Rep. 389 263440 (2004).

[36] In principle there is nothing wrong with $\alpha^{Q}>1$ evaluations (although we note that for pure "neutron" matter the quark phase asymmetry should be 1 , since $\rho_{d}=2 \rho_{u}$ ). Indeed for low $\chi$-values, $\chi<0.1$, very small quark concentrations, we can get $\alpha^{Q}$ values slightly larger than 1. However we are cautious about these results since we can expect also some numerical problems. In fact for very small $\chi$ values the weight of the $\alpha^{Q}$ contribution in the minimization procedure is expected not too relevant, as we can clearly see from the Eq.(8). In any case the important point is that this is not affecting the general discussion about the isospin distillation.

[37] A.N. Sissakian, A.S. Sorin, V.D. Toneev, Phys.Part.Nucl. 391062 (2008).

[38] J.B. Kogut, D.K. Sinclair, Phys.Rev. D70 095401 (2004).

[39] D. Toublan, J.B. Kogut, Phys.Lett. B605 129 (2005).

[40] C. Fuchs, H. Lenske, H.H. Wolter, Phys.Rev. C52 3043 (1995).

[41] S. Typel, H.H. Wolter, Nucl.Phys. A656 331 (1999).

[42] S.S. Avancini, L. Brito, D.P. Menezes, C. Providencia, Phys.Rev. C70 015203 (2004).

[43] F. Hofmann, C.M. Keil, H. Lenske, Phys.Rev. C64 034314 (2001).

[44] P. Goegelein, E.N.E. van Dalen, C. Fuchs, H. Müther, Phys.Rev. C77 025802 (2008).

[45] B. Liu et al., Phys.Rev. C75 048801 (2007).

[46] M. Frank, M. Buballa, M. Oertel, Phys.Lett. B562 221 (2003).

[47] Guo-yun Shao et al., Phys.Rev. D73 076003 (2006).

[48] S. Plumari, Ph.D.Thesis, 2009 Univ.Catania.

[49] N. Kaiser, W. Weise, Phys.Lett. B671 25 (2009).

[50] W. Weise, Prog.Theor.Phys.Suppl. 170161 (2007).

[51] M.G. Alford, A. Schmitt, K. Rajagopal, T. Schafer, Rev.Mod.Phys. 801455 (2008).

[52] Mei Huang, Pengfei Zhuang, Weiqin Chao, Phys.Rev. D67 065015 (2003).

[53] H. Stöecker, private communication.

[54] G. Ferini, M. Colonna, T. Gaitanos, M. Di Toro, Nucl.Phys. A762 147 (2005).

[55] L. Csernai, D. Rohrich, Phys.Lett. B458 454 (1999).

[56] R.J. Fries, V. Greco V, P. Sörensen, Ann.Rev.Nucl.Part.Sci. 58177 (2008).

[57] V. Greco, M. Colonna, M. Di Toro, F. Matera, Phys.Rev. C67 015203 (2003).

[58] B. Liu, H. Guo, M. Di Toro, V. Greco, Eur.Phys.J. A25 293 (2005).

[59] T. Gaitanos, M. Colonna, M. Di Toro, H.H. Wolter, Phys.Lett. B595 209 (2004).

[60] T. Klähn et al., Phys.Rev. C74 035802 (2006). 